Chemical Physics Letter (2003), accepted

\title{
Characterization of single-walled carbon nanotubes catalytically synthesized from alcohol
}

\author{
Yoichi Murakami, Yuhei Miyauchi, Shohei Chiashi, Shigeo Maruyama*
}

Department of Mechanical Engineering, The University of Tokyo

7-3-1 Hongo, Bunkyo-ku, Tokyo 113-8656, Japan

Received 1 March 2003; in final form 1 April 2003

\begin{abstract}
High purity single-walled carbon nanotubes (SWNTs) were synthesized from ethanol by catalytic CVD method. The yield of SWNTs was determined based on TGA complemented by Raman and TEM analyses. The effects of CVD reaction time and pre-reduction of catalytic metal on the yield and quality of synthesized SWNTs were investigated. The SWNT yield of more than $40 \%$ was achieved over the weight of zeolite support powder with Fe/Co catalyst, which corresponded to more than $800 \%$ yield over the weight of the catalytic metal, within the CVD reaction time of 120 minutes assuring as-grown high quality.
\end{abstract}

\section{Introduction}

Since the discovery by Iijima and Ichihara [1], single-walled carbon nanotubes (SWNTs) have been extensively studied due to their outstanding potentials as a future material. To present, their unique properties have evoked several novel applications [2] that would never be possible with

\footnotetext{
* Corresponding Author. Fax: +81-3-5800-6983.

E-mail address: maruyama@photon.t.u-tokyo.ac.jp (S. Maruyama).
} 
conventional materials, and vast rooms are left for such inventions using SWNTs. With this concern, a synthesis technique of SWNTs with a higher quality and at a lower cost is an urgent priority at present.

In addition to the initial laser-furnace [3] and arc-discharge [4] techniques, a variety of studies were reported [5-14] using chemical vapor deposition (CVD) technique that is suitable to mass production. Many kinds of carbon sources, support materials, and synthesis conditions have been tested so far tested, but the best approach has not been set up yet. Aiming at this common goal, our group reported [15] that SWNTs with remarkable purity was synthesized at temperature as low as $550{ }^{\circ} \mathrm{C}$ using alcohol (ethanol and methanol). Further merits of using alcohol lie in its harmless, low cost, and easy-handling nature.

There is no report that supported their validity of the SWNT yield by a simultaneous exhibition of Raman, TGA, and TEM results, other than that by Alvarez et al. [12]. Furthermore, in most of the reports the characters for their reportedly highest yield were not presented at all. It is authors' belief that sufficient discussion should be made on the definition and presentation of the yield of SWNTs, with an explicit notation of the upper yield limit for practical quality. In this report, the effects of CVD reaction time and pre-reduction of catalytic metal on the quality and yield of synthesized SWNTs have been investigated to discuss the feasibility toward mass production of our CVD method from alcohol. 


\section{Experiment and measurement}

We employed iron and cobalt for catalytic metals and USY-zeolite powder (over $99 \% \mathrm{SiO}_{2}$ ) for their support $[16,17]$ to form catalytic powder. See Ref. [15] for detailed procedure for preparation. The concentration of each metal species in the catalytic powder was $2.5 \mathrm{wt} \%$.

In the CVD procedure, $33 \mathrm{mg}$ of the catalytic powder was sparsely placed on a quartz boat, which was then placed at the center of a quartz tube (26 mm inner diameter, $1 \mathrm{~m} \mathrm{long})$. The central $30 \mathrm{~cm}$ of the quartz tube was heated up with an electric furnace, flowing more than $200 \mathrm{sccm}$ of $\mathrm{Ar}$ so that the pressure inside the tube was kept about 300 Torr. After the reaction temperature was achieved typically in 30 minutes, Ar supply was stopped and inside of the quartz tube was evacuated by a rotary pump. Subsequently, ethanol vapor was introduced from a temperature-controlled reservoir, keeping the ethanol pressure measured just before the entrance of the quartz tube constant. Finally, the ethanol supply was stopped and the furnace was turned off before bringing the system back to room temperature with flowing $100 \mathrm{sccm}$ of Ar. In some experiments, Ar containing $3 \%$ of hydrogen (referred to as ' $\mathrm{Ar} / \mathrm{H}_{2}$ ') was used for a reduction purpose, instead of pure Ar.

'As-grown' blackened powder was characterized by thermo-gravimetric analysis (TGA), Raman spectroscopy, and transmission electron microscopy (TEM). TGA was performed with Seiko Instruments Inc. Exter 6000 and TG/DTA 6300, and with a heating rate of $5{ }^{\circ} \mathrm{C} / \mathrm{min}$ with $100 \mathrm{sccm}$ flow of air. In every TGA measurement, the sample of $10 \pm 1 \mathrm{mg}$ was used in order to suppress TG error below $1 \%$, and it was held at $100{ }^{\circ} \mathrm{C}$ for $2 \mathrm{~h}$ before starting to raise the temperature to ensure sufficient reproducibility. Raman measurement was performed with CHROMEX 501 is and ANDOR Technology DV401-FI for the spectrometer and CCD system, respectively, with an optical system of SEKI TECHNOTRON Corp.'s STR250. Laser wavelength of $488 \mathrm{~nm}$ was used in all the 
measurements.

\section{Definition of SWNT yield}

We utilized recent findings about TG or TPO (temperature-programmed oxidation) analyses $[10,12,18-22]$ for the estimation of SWNT yield. The burning temperature of SWNTs is largely influenced by the condition of the SWNTs specimen, so caution must be taken in comparing these results. Most remarkably, when catalytic metal particles (or metallic impurity) are mixed among SWNTs, the decomposition temperature is somewhat lowered from that of pure SWNTs [18-21]. At least, the burning temperatures of purified and annealed SWNTs produced by laser furnace method $[3,19]$ or by HiPco method $[8,20,21]$ are in the same temperature range of $500 \sim$ $700{ }^{\circ} \mathrm{C}$.

Since the amorphous carbon decomposes around $300 \sim 500{ }^{\circ} \mathrm{C}[10,12,18]$, which is lower than the decomposition temperature of SWNTs, the existence of amorphous carbon is readily recognized from TG or TPO curves. However, when graphitized carbon products such as MWNTs coexist with SWNTs, it becomes rather difficult to specify the amount of SWNTs only by TGA or TPO. This is because, as pointed out by Alvarez et al. [12], some kind of well-graphitized product also burns at higher temperature range that overlaps the range of SWNTs. As a possible method for estimating the amount of each constituent, Alvarez et al. [12] decomposed their TPO curves into several Gaussian-Lorentzian components and attempted to estimate the amount of each carbon species including amorphous carbons and MWNTs. However, this method accompanies an abundant freedom in the choice of fitting parameters; hence it may not be the best way, though physically effective, for an accurate estimation of the yield.

The safest way is, therefore, to perform the estimation only taking the target specimen that 
contains hardly any amorphous carbon nor MWNTs, for which no Gaussian-Lorentzian decomposition is essentially needed: It is possible to confirm the absence of amorphous carbon by TGA and that of MWNTs by TEM. The yield of SWNTs in this report is defined as the ratio of weight loss between 500 and $700{ }^{\circ} \mathrm{C}$ to the weight remained at $1000{ }^{\circ} \mathrm{C}$, both measured in TGA, which is a representative ratio of the weight gain by SWNTs to the weight of the catalytic powder.

\section{Results and discussion}

Figure 1(a) shows the TG and DTG curves of the 'as-prepared' powders for various CVD reaction times when Ar was used while heating-up of the electric furnace. The reaction temperature and pressure were $800{ }^{\circ} \mathrm{C}$ and 10 Torr, respectively. This temperature had been confirmed by Raman and TEM analyses to be optimal (i.e. achieving minimum impurities) in this condition. In our previous report [15] SWNTs with sufficiently high quality/purity were synthesized even at $650{ }^{\circ} \mathrm{C}$, but this was possible because the pressure was much lower $(2 \sim 5$ Torr $)$ than that in the present study, where potentials for the highest yield is discussed. A slight weight gain around $250{ }^{\circ} \mathrm{C}$ is due to oxidation of catalytic metals [20,21], and the following weight loss up to $500{ }^{\circ} \mathrm{C}$ is due to burning of amorphous carbons. We assigned the weight loss between 500 and $700{ }^{\circ} \mathrm{C}$ to burning of SWNTs, which strongly depends on the CVD reaction time. Note that the burning temperature of current SWNTs is equal to that of purified and annealed SWNTs synthesized by the laser-furnace [19] and HiPco [20, 21] methods, indicating that the sidewalls of our SWNTs are of good quality and are free from metal particles. The gradual shift of DTG peaks toward higher temperature is attributed to the diameter increase of SWNTs or thickening of the bundles. For the 300-min case, this shift is partially due to the formation of MWNTs. According to TEM observations, no multi-layered carbons such as MWNTs were observed up to the 120-min case, 
while small amount of MWNTs were observed in the 300-min case (Not shown).

A series of experiments were carried out using $\mathrm{Ar} / \mathrm{H}_{2}$ and their TG and DTG results are presented in Fig. 1(b). The ethanol pressure during the reaction was 10 Torr. The first experiment was made under the condition of $800{ }^{\circ} \mathrm{C}, 10 \mathrm{~min}$ for comparison with the case of Fig. 1(a), where Ar was used. The TG curve in the case of $\mathrm{Ar} / \mathrm{H}_{2}$ (dashed line) exhibits a larger amount of total carbon yield than the case of Ar (Fig. 1(a)): This increase is thought to emanate from an additional reduction of catalytic metals that would be otherwise reduced by carbon atoms in a slower speed. A side effect, i.e., simultaneous increase of amorphous carbon, can be suppressed by employing higher reaction temperature. The solid lines in Fig. 1(b) represent the cases synthesized at $850{ }^{\circ} \mathrm{C}$, showing that the amount of amorphous carbon was suppressed to the extent of Fig. 1(a). Note that this comparison means not only that $\mathrm{Ar} / \mathrm{H}_{2}$ enhances the yield of SWNTs but also that Ar lowers the reaction temperature, which can be an important controllable parameter in the SWNT production. A remarkable difference in the yields indicates that the reduction by carbon atoms is a reaction of a long time-scale, extending to several tens of minutes or longer, as shown later in Fig. 3. Alvarez et al. [12] presented their detailed investigation on the degree of reduction in their bimetallic $\mathrm{Mo} / \mathrm{Co}$ catalyst, showing that even after 30 minutes of reduction with $\mathrm{H}_{2}$ at $500{ }^{\circ} \mathrm{C}$, the metal was still in some part oxidized.

The Raman spectra corresponding to Fig. 1(b) are presented in Fig. 2. Detailed interpretations of the Raman spectra are presented elsewhere [23]. The decrease in the D-band magnitude from '10 min @ $800{ }^{\circ} \mathrm{C}$ ' to '10 min @ $850{ }^{\circ} \mathrm{C}$ ' coincides the observation in Fig. 1(b). Although the magnitudes of the D-band gradually increased with the reaction time, they still retained sufficiently good quality according to TEM observations. Since the TGA results indicate a negligible amount of amorphous carbon compared with SWNTs even in the longer time cases, the D-band increase in this case may partly be ascribed to the double resonance effect by SWNTs with 
defects. The left panel shows the radial breathing mode (RBM) of SWNTs, from which the diameter distribution of SWNTs can be estimated using a relationship ' $d(\mathrm{~nm})=248 / v\left(\mathrm{~cm}^{-1}\right)$ ' $[24,25]$, where $d$ is diameter of a SWNT and $v$ is the Raman shift. The observed increases in the diameter distribution and D-band magnitude with CVD reaction time are partly due to the fact that thinner nanotubes are more difficult to survive through the long reaction exposure, at the same time the thicker nanotubes, which are harder to form a cap and get properly annealed [6], are faster to grow in a long run.

The time progresses of the SWNT yields are plotted in Fig. 3. The error arising from TG was $\pm 0.5 \%$. Note that the definition of SWNT yield is not strictly applicable for the cases of 300 minutes with Ar, where MWNTs were observed. The qualities of as-grown SWNTs were confirmed by TEM, and some of the representative images are presented in Fig. 4. As for the achievement of the yield and quality, the cases of ' 10 Torr, $60 \mathrm{~min}, \mathrm{Ar} / \mathrm{H}_{2}$ ' (Fig. 4(a)) and ' 5 Torr, $60 \mathrm{~min}$, Ar' exhibit the highest quality. The quality of ' 10 Torr, $120 \mathrm{~min}, \mathrm{Ar} / \mathrm{H}_{2}$ ' was nearly the same (Fig. 4(b)) but had thicker bundles, and that of ' 10 Torr, 60 min, Ar' followed them. In this study, therefore, the practical limit of the reaction time to assure sufficient quality/purity of the product is $120 \mathrm{~min}$, in which SWNT yield of more than $40 \%$ was achieved over the weight of the zeolite support powder with $\mathrm{Fe} / \mathrm{Co}$ catalyst. This corresponds to more than $800 \%$ yield over the weight of the catalytic metal, since the total metallic weight in the catalytic powder was $5 \%$. Note that the 'as-grown' high quality of our SWNTs is apparent when the current TEM pictures (Fig. 4) are compared with those presented in previous reports, for example $[8-12,14,20]$. Our choice of ethanol for a carbon feedstock seems to be the crucial point.

\section{Concluding remarks}


We have demonstrated that more than $40 \%$ yield of SWNTs with sufficiently high quality can be synthesized from ethanol within the CVD time of $120 \mathrm{~min}$, with which the feasibility of proposed method toward mass production has been presented. The reduction of catalytic metal, which leads to effective enhancement of the yield, plays an important role in the synthesis of SWNTs. Further optimization of catalytic metal species and support material is needed along with an elucidation of the growth mechanism to achieve still higher quality and productivity of SWNTs.

\section{Acknowledgements}

Authors express cordial thanks to Mr. H. Tsunakawa, Mr. T. Ito, and Mr. Y. Kakegawa for their assistances in TEM observations. Part of this work was financially supported by Grant-in-Aid \#12450082, 13555050, and 13GS0019, from Japan Ministry of Education, Culture, Sports, Science and Technology. 


\section{References}

[1] S. Iijima, T. Ichihara, Nature 363 (1993) 603.

[2] M. S. Dresselhaus, G. Dresselhaus, P. Avouris, Carbon Nanotubes, Springer, Berlin, 2001.

[3] A. Thess, R. Lee, P. Nikolaev, H. Dai, P. Petit, J. Robert, C. Xu, Y. H. Lee, S. G. Kim, A. G. Rinzler, D. T. Colbert, G. E. Scuseria, D. Tománek, J. E. Fischer, R. E. Smalley, Science 273 (1996) 483.

[4] C. Journet, W. K. Maser, P. Bernier, A. Loiseau, M. L. de la Chapelle, S. Lefrant, P. Deniard, R. Lee, J. E. Fisher, Nature 388 (1997) 756.

[5] H. Dai, A. G. Rinzler, P. Nikolaev, A. Thess, D. T. Colbert, R. E. Smalley, Chem. Phys. Lett. $260(1996) 471$.

[6] J. H. Hafner, M. J. Bronikowski, B. R. Azamian, P. Nikolaev, A. G. Rinzler, D. T. Colbert, K. A. Smith, R. E. Smalley, Chem. Phys. Lett. 296 (1998) 195.

[7] J. Kong, A. M. Cassell, H. Dai, Chem. Phys. Lett., 292 (1998) 567.

[8] P. Nikolaev, M. J. Bronikowski, R. K. Bradley, F. Rohmund, D. T. Colbert, K. A. Smith, R. E. Smalley, Chem. Phys. Lett. 313 (1999) 91.

[9] J.-F. Colomer, J.-M. Benoit, C. Stephan, S. Lefrant, G. Van Tendeloo, J. B. Nagy, Chem. Phys. Lett. 345 (2001) 11.

[10] B. Kitiyanan, W. E. Alvarez, J. H. Harwell, D. E. Resasco, Chem. Phys. Lett., 317 (2000) 497.

[11] M. Su, B. Zheng, J. Liu, Chem. Phys. Lett. 322 (2000) 321.

[12] W. E. Alvarez, B. Kitiyanan, A. Borgna, D. E. Resasco, Carbon, 39 (2001) 547.

[13] H. Yan, Q. Li, J. Zhang, Z. Liu, Carbon, 40 (2002) 2693.

[14] B. Zheng, Y. Li, J. Liu, Appl. Phys. A, 74 (2002) 345.

[15] S. Maruyama, R. Kojima, Y. Miyauchi, S. Chiashi, M. Kohno, Chem. Phys. Lett., 360 (2002) 229. 
[16] K. Mukhopadhyay, A. Koshio, N. Tanaka, H. Shinohara, Jpn. J. Appl. Phys. 37 (1998) L1257.

[17] K. Mukhopadhyay, A. Koshio, T. Sugai, N. Tanaka, H. Shinohara, Z. Konya, J. B. Nagy, Chem. Phys. Lett. 303 (1999) 117.

[18] E. Mizoguti, F. Nihey, M. Yudasaka, S. Iijima, T. Ichihashi, K. Nakamura, Chem. Phys. Lett., $321(2000) 297$.

[19] I. W. Chiang, B. E. Brinson, R. E. Smalley, J. L. Margrave, R. H. Hauge, J. Phys. Chem. B, $105(2001) 1157$.

[20] W. Zhou, Y. H. Ooi, R. Russo, P. Papanek, D. E. Luzzi, J. E. Fischer, M. J. Bronikowski, P. A. Willis, R. E. Smalley, Chem. Phys. Lett. 350 (2001) 6.

[21] M. Chike, J. Li, B. Chen, A. Cassell, L. Delzeit, J. Han, M. Mayyappan, Chem. Phys. Lett. 365 (2002) 69.

[22] M. Zhang, M. Yudasaka, A. Koshio, S. Iijima, Chem. Phys. Lett., 364 (2002) 420.

[23] M. S. Dresselhaus, P. C. Eklund, Advances in Physics, 49 (2000) 705.

[24] R. Saito, G. Dresselhaus, M. S. Dresselhaus, Phys. Rev. B 61 (2000) 2981.

[25] A. Jorio, R. Saito, J. H. Hafner, C. M. Lieber, M. Hunter, T. McClure, G. Dresselhaus, M. S. Dresselhaus, Phys. Rev. Lett. 86 (2001) 1118. 


\section{Figure Captions}

Fig. 1. TG/DTG curves when (a) $\mathrm{Ar}$ and (b) $\mathrm{Ar} / \mathrm{H}_{2}$ were used during the heat-up of the electric furnace. The ethanol pressure during CVD reaction was 10 Torr in all cases. The reaction temperature in panel (a) was $800{ }^{\circ} \mathrm{C}$.

Fig. 2. Raman spectra corresponding to Fig. 1(b). The left panel is a magnification of the low-frequency area of the right. The gas used in the heating-up of the electric furnace and the ethanol pressure during CVD reaction were $\mathrm{Ar} / \mathrm{H}_{2}$ and 10 Torr, respectively.

Fig. 3. Time progresses of SWNT yields for the conditions of ' $850{ }^{\circ} \mathrm{C}, 10 \mathrm{Torr}, \mathrm{Ar} / \mathrm{H}_{2}$ ' (circle), ' 800 ${ }^{\circ} \mathrm{C}, 10$ Torr, Ar' (square), and ' $800{ }^{\circ} \mathrm{C}, 5$ Torr, Ar' (diamond), for the temperature, pressure, and gas used in the heating-up of the electric furnace, respectively. A straight line for each condition denotes a least-squares fit. Note that a small amount of multi-layered carbon products were observed in cases of 300 minutes.

Fig. 4. TEM images for 'as-grown' SWNTs synthesized at the conditions of (a) $850{ }^{\circ} \mathrm{C}, 10$ Torr, 60 $\min , \mathrm{Ar} / \mathrm{H}_{2}$, and (b) $850{ }^{\circ} \mathrm{C}, 10 \mathrm{Torr}, 120 \mathrm{~min}, \mathrm{Ar} / \mathrm{H}_{2}$, for the temperature, pressure, time, and gas used during the heat-up of the electric furnace, respectively. Note the high purity of SWNTs without contaminants such as metallic particles, amorphous carbons, or MWNTs. 


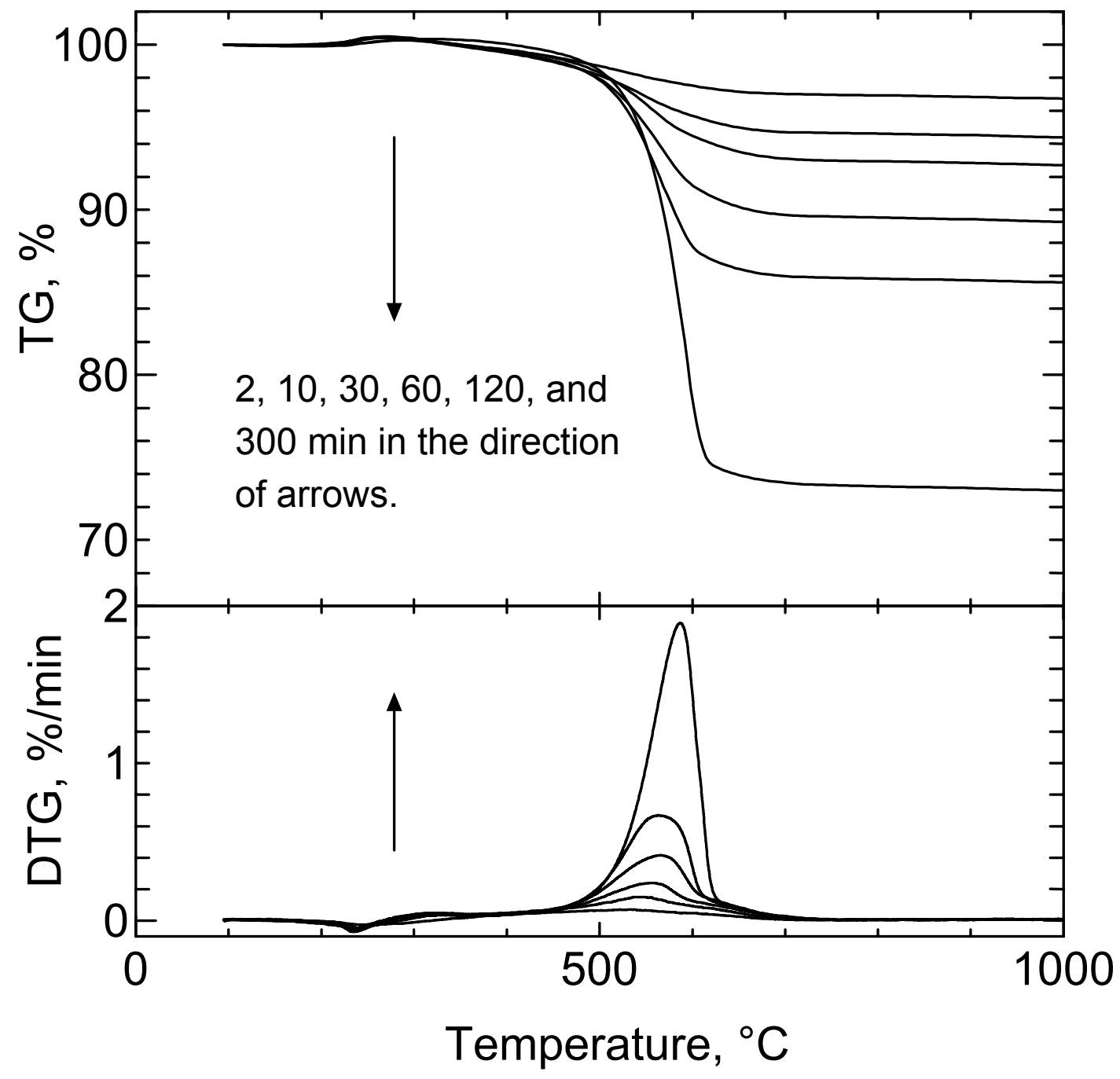

Fig. 1(a) 


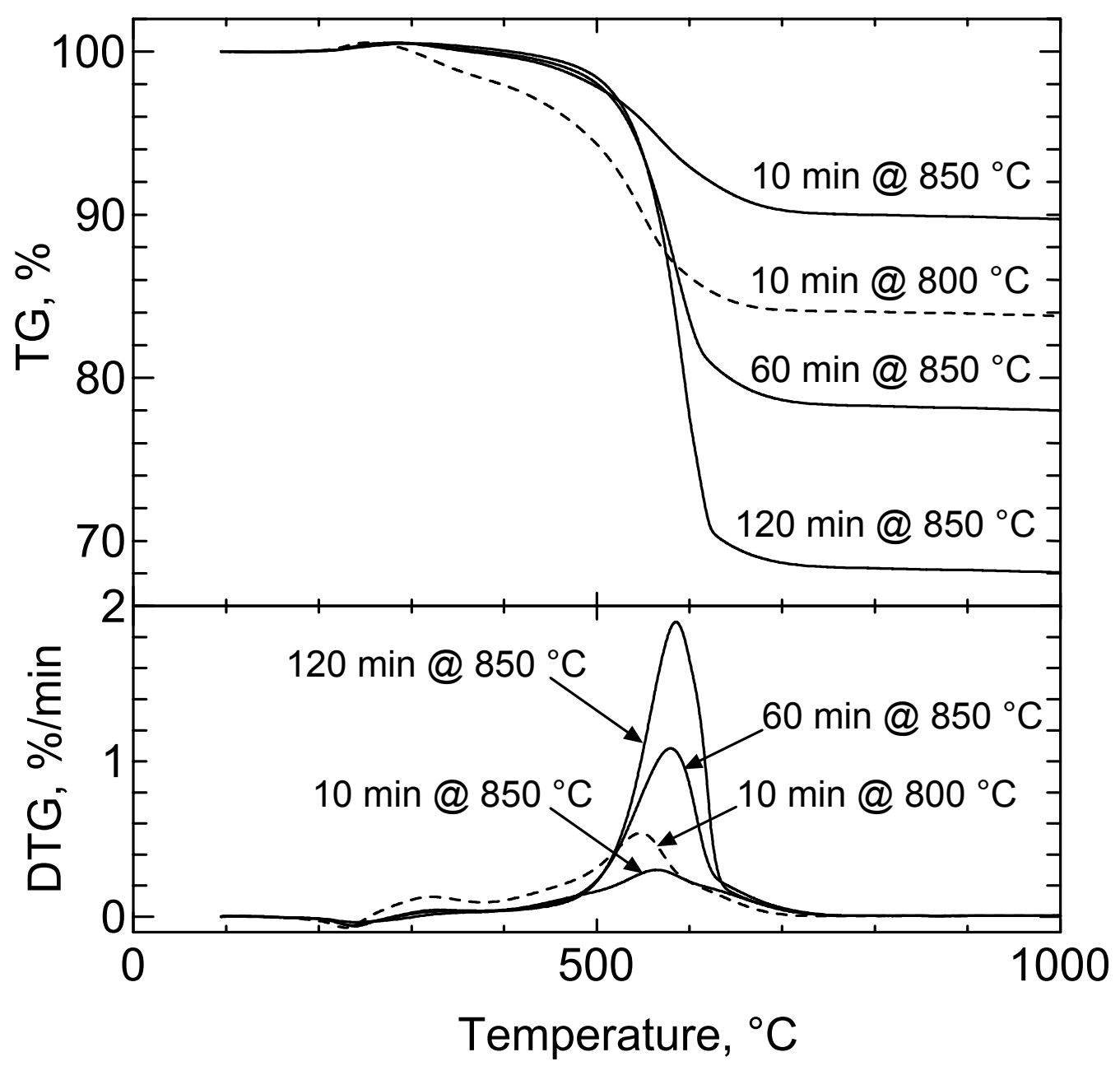

Fig. 1(b) 


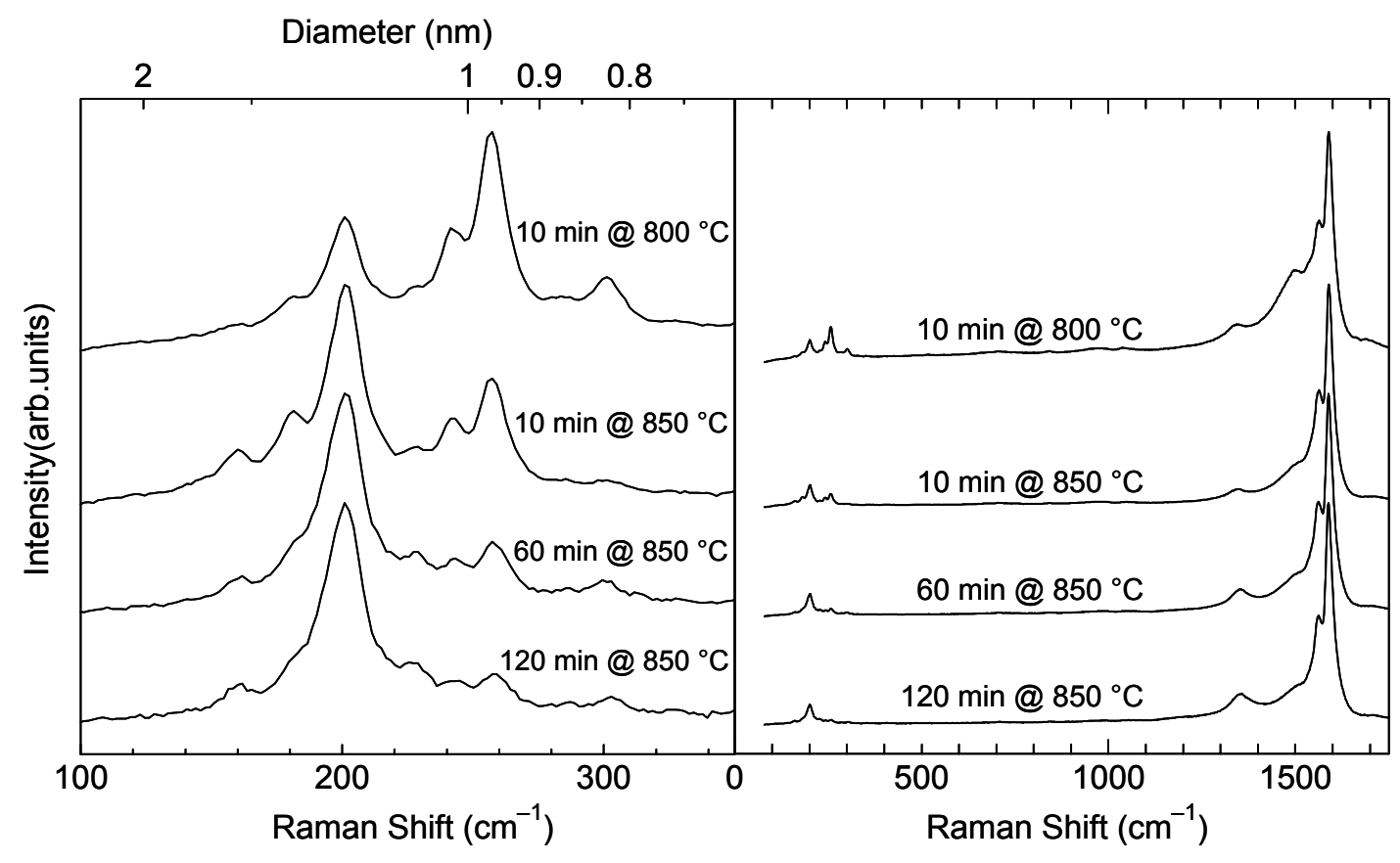

Fig. 2 


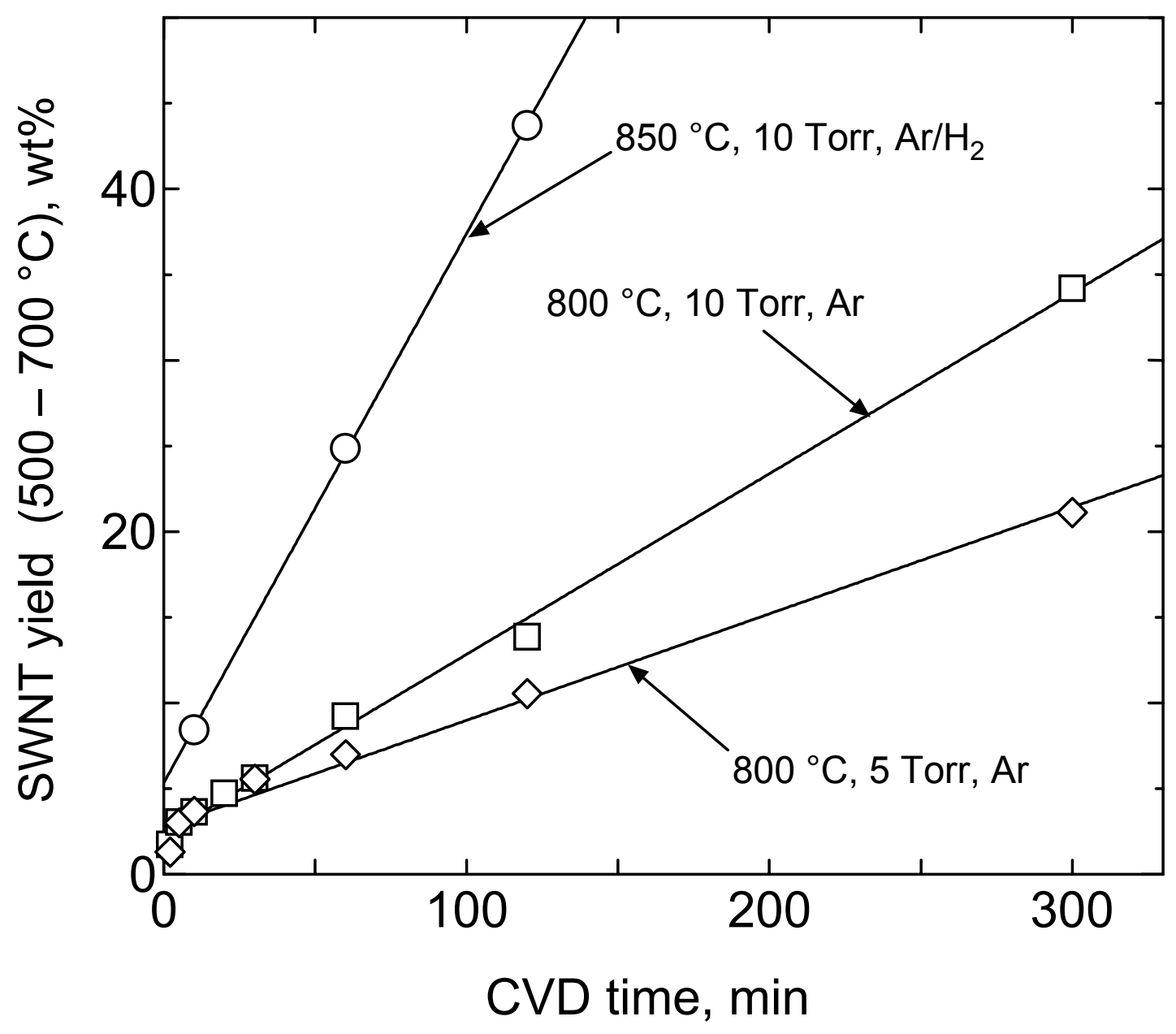

Fig. 3 


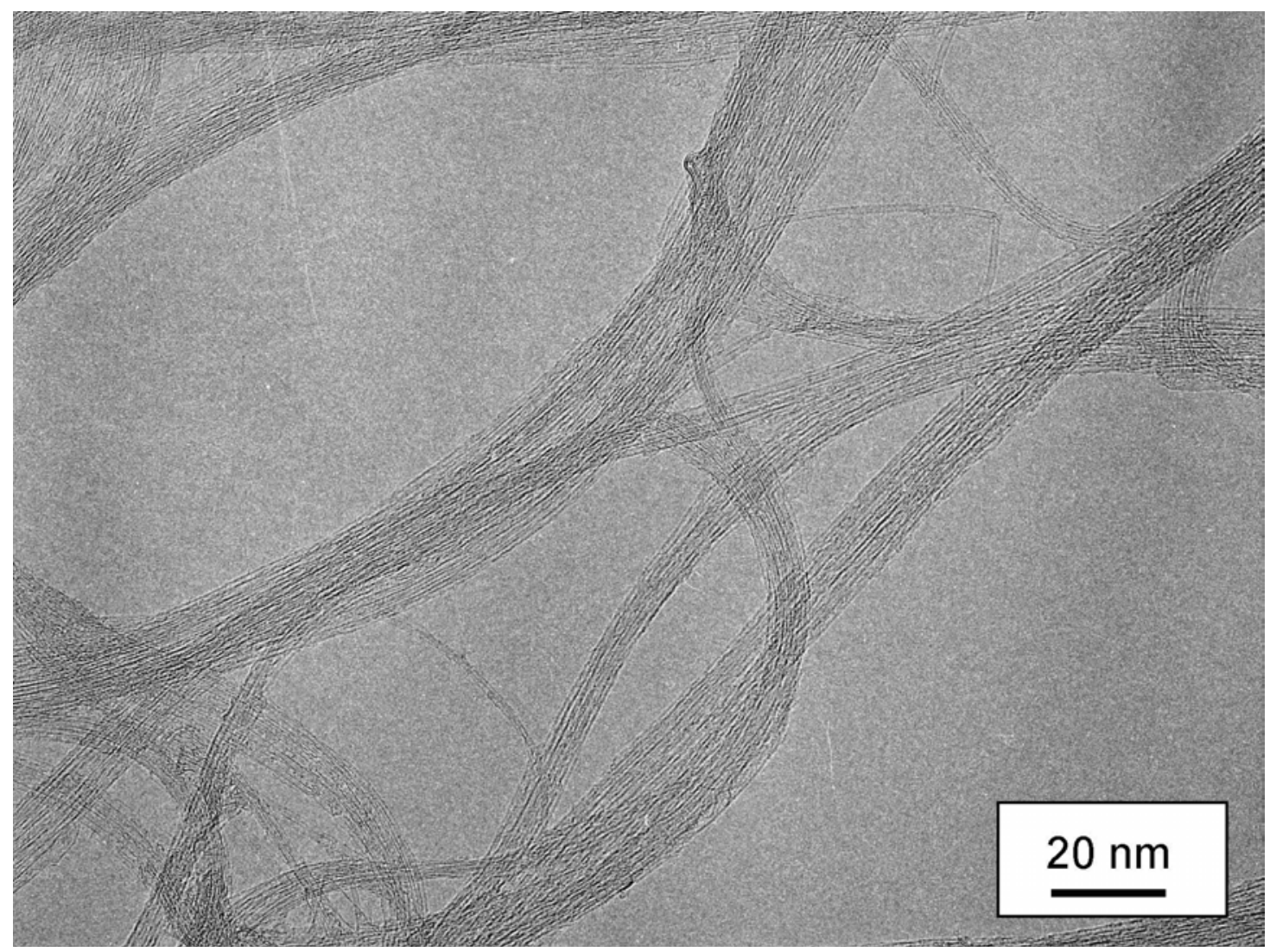

Fig. 4 (a) 


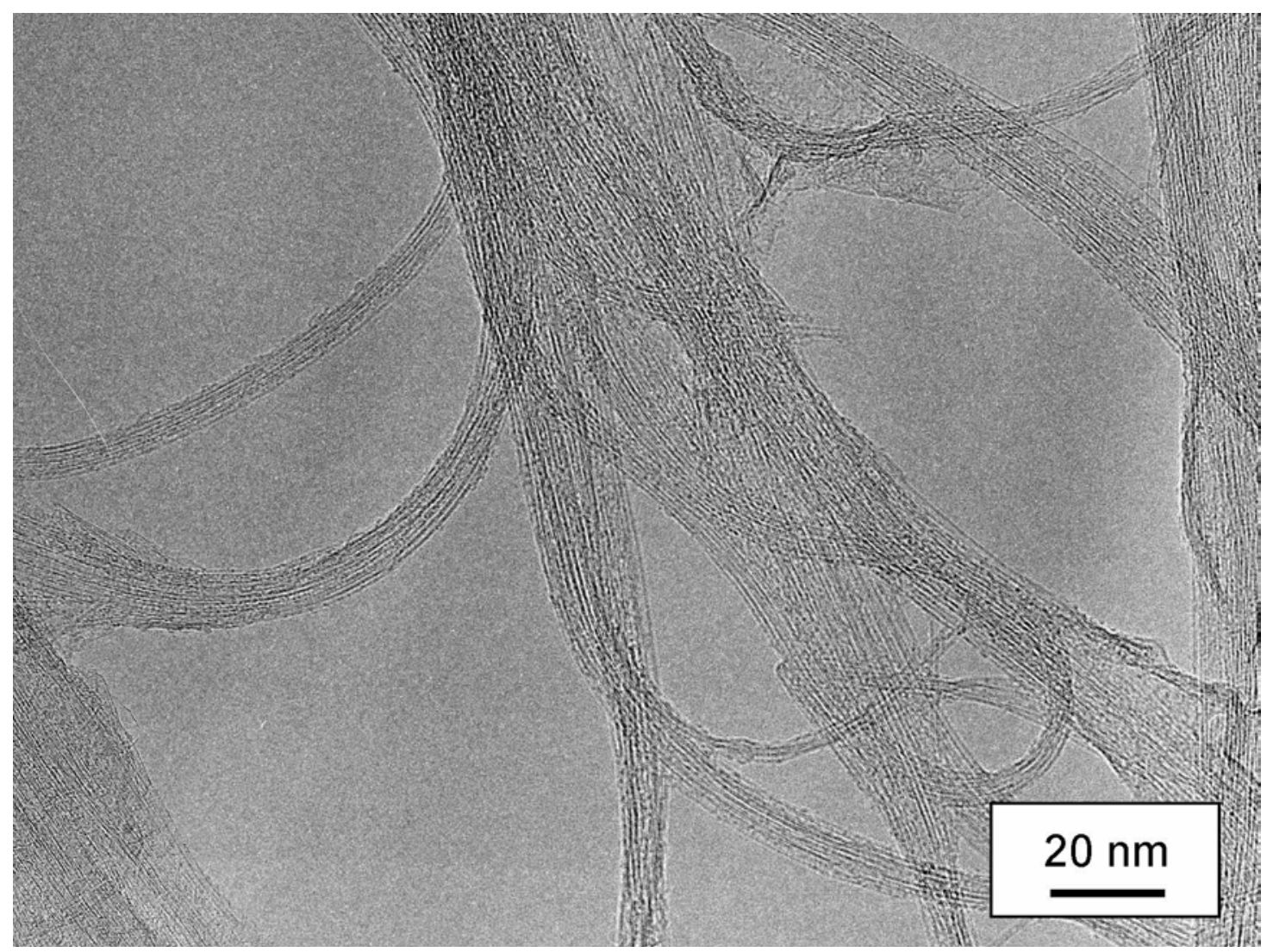

Fig. 4 (b) 